\title{
Discrete-Velocity Simulations of High-speed Flows Based on Binary Gas Mixture Kinetic Models
}

\author{
Blaga N. Todorova ${ }^{1, b)}$, Craig White ${ }^{1, c)}$ and René Steij $1^{1, a)}$ \\ ${ }^{1}$ School of Engineering, University of Glasgow, Glasgow, United Kingdom

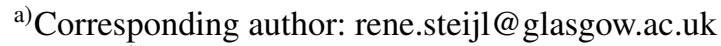 \\ b)b.todorova.1@research.gla.ac.uk \\ ${ }^{c)}$ craig.white.2@glasgow.ac.uk
}

\begin{abstract}
Many high-speed flows of engineering and scientific importance involve gas mixtures. Accounting for the species diffusion in such flows is essential when strong species gradients and temperature gradients occur. The domain of possible applications includes hypersonic rarefied gases, chemical reacting flows and plasmas. Kinetic models for multicomponent gases have been considered since the original BGK model was formulated. However, BGK-derived models pose a number of difficulties, e.g. avoiding negative density and temperatures. A distinct challenge of the BGK approximation lies in the number of correct transport coefficients recovered in the continuum limit. Two new kinetic models- a Shakhov-based model and an ES-based model- were recently introduced. Both methods are capable of modelling a binary mixture of monoatomic gases and account for separate species-mean velocity such that the species diffusion and velocity drift are accurately represented. The main advantage is the recovery of three correct transport coefficients in the hydrodynamic limit and fixing the Prandtl number. The resulting models are implemented in a parallel multi-block discrete-velocity solver and applied to a range of test cases. This paper shows the solutions of the models for a variety of high-speed flows, obtained using the discrete-velocity method. The models are first validated against known results for normal shocks, showing good agreement for species density and temperature profiles. The models are evaluated for more demanding test cases involving flows under different conditions around a circular cylinder. A detailed comparison with DSMC results demonstrates promising results from both kinetic models.
\end{abstract}

\section{INTRODUCTION}

The Navier-Stokes equations lose their validity in the rarefied regime and a more detailed level of flow modelling for applications like hypersonic rarefied gases, chemical reacting flows and plasmas is required. This can be provided by the Boltzmann equation [1], since it is capable of modelling non-equilibrium flows, mixtures of monoatomic and diatomic gases and also flows with or without chemistry. However, the collision integral of the equation is highly complex and computationally unfeasible for most applications of interest. For that reason, Boltzmann relaxation models were introduced, but their capabilities are limited and still represent an area of active research. Current techniques include the statistical Monte Carlo method [2] that can be problematic in the transitional regime. Alternatively, the discrete velocity method and UGKS [3] and DUGKS [4] schemes are numerical methods, which apply kinetic models. However, there is a need for further improvements on the kinetic models for gas mixtures.

There are two main categories of BGK models that deal with gas mixtures. Depending on the treatment of the collision term, models are classified as single-relaxation models, e.g. [5, 6, 7, 8] and multi-relaxation models, e.g. [9, 10, 11]. The multi-relaxation models allow treating self- and cross-collisions with different relaxation rates, while the collisions of the single-relaxation models are treated with the same relaxation rate. This suggests multi-relaxation models are advantageous for the modelling of multi-species gases, specifically when the species differ significantly from each other, e.g. high mass ratio. However, important advantages the single-relaxation models provide are the simplicity and computational efficiency. For this reason, we focus our attention at studying single-relaxation models and evaluating their capabilities.

The first consistent single relaxation kinetic model was introduced by Andries, Aoki and Perthame [5]. Three further developed models- Brull, Pavan and Schneider's BGK mixture [6], Brull's ES-BGK mixture [7] and Groppi, Monica and Spiga's [8] models were established. Common characteristic for these models is that the simplified collision 
term of the Boltzmann equation contains one relaxation term, which captures interspecies collisions as well as selfcollisions. This collision frequency is not dependent on velocity, but only on the viscosity and pressure of the mixture. Other good mathematical properties of the models include the reduction of the distribution function to a Maxwellian distribution in equilibrium and the conservation of mass, momentum and energy. One of the biggest challenges for inert monoatomic gas mixtures is the correct fit of transport coefficients in the continuum limit. Andries et al.'s model recovers the correct viscosity,Brull et al.'s BGK mixture model has the correct Fick and Newton's laws as does the Groppi et al.'s model. The ES-BGK mixture model by Brull recovers the correct viscosity and Fourier coefficient. We have previously introduced two new kinetic models by building upon the Groppi et al.'s model and incorporating a Shakhov-based and ES-based corrections [12]. The models inherit the described mathematical properties and the correct ordinary diffusion and viscosity coefficients of the Groppi et al.'s model and addresses the thermal conductivity coefficient. The key contribution of these two models is the correct fit of three transport coefficients- Fick, Newton and Fourier laws, which also leads to a correct Prandtl number.

The resulting models are implemented in the parallel multi-block discrete-velocity solver within the MФC framework $[13,14]$ and applied to a range of test cases. The models were previously validated against known results for normal shocks for a binary gas mixture, since it's the simplest form of high non-equilibrium flow and is well inspected. First, we present a comparison between the Groppi, Shakhov-based and ES-based corrections with results from the full Boltzmann equation [15]. By introducing a study of the total heat flux through the shock, the significance of the new kinetic models is emphasized. The more complex flow around a circular cylinder is then considered and a parametric study shows the change in the flow-field when the temperature of the solid is varied and when the mass ratio between species is increased. The Shakhov-based and ES-based models are compared with results for neon-argon and neonkrypton mixtures, using DSMC [16]. The numerical results demonstrate good agreement with validation data and are promising for engineering applications that include gas mixtures.

The rest of the paper is organized as follows. The next section summarizes the key points of the two newly introduced Shakhov-based and ES-based models for a binary monoatomic gas mixture. The focus of this paper is the application of the two models and is split between the numerical results for a normal shock wave and the results for a circular cylinder. The last part of the paper includes concluding remarks and future work.

\section{KINETIC MODELS}

\section{Shakhov-based Model}

For a binary gas mixture the governing equation is per species $s=1,2$ (Equation 1) and the simplified collision operator $Q_{s}$ involves the Shakhov-based [17] mixture distribution function $G_{s}^{s h}$ and a common collision rate $v$.

$$
\frac{\partial f_{s}}{\partial t}+\underline{u} \cdot \frac{\partial f_{s}}{\partial \underline{x}}=Q_{s} \quad ; \quad Q_{s}=v\left(G_{s}^{s h}-f_{s}\right) ; \quad ; \quad s=1,2
$$

From the species macroscopic variables, the overall gas mixture properties are obtained as shown in Equation 2.

$$
\begin{gathered}
\sum_{s=1}^{2} n_{s}=n ; \quad \sum_{s=1}^{2} \rho_{s}=\rho ; \sum_{s=1}^{2} \rho_{s} \underline{u}_{s}=\rho_{0} \underline{u}_{0} ; \sum_{s=1}^{2} \rho_{s}\left(\underline{u}_{s}-\underline{u}_{0}\right)^{2}=\sum_{s=1}^{2} \rho_{s}\left|\underline{u}_{s}\right|^{2}-\rho\left|\underline{u}_{0}\right|^{2} \\
\frac{3}{2} n k T=\sum_{s=1}^{2} \frac{3}{2} n_{s} k T_{s}+\frac{1}{2} \sum_{s=1}^{2} \rho_{s}\left(\underline{u}_{s}-\underline{u}_{0}\right)^{2}
\end{gathered}
$$

The distribution function $G_{s}^{S h}$ is in the form:

$$
G_{s}^{S h}(\underline{u})=G_{s}(\underline{u})\left\{1+\frac{2(1-\operatorname{Pr})\left(\underline{u}-\underline{u}_{s}^{(g)}\right) \cdot \underline{q}_{s}^{\text {corr }}}{5 p_{s} k \hat{T} / m_{s}}\left[\frac{m_{s}}{2 k \hat{T}}\left(\underline{u}-\underline{u}_{s}^{(g)}\right)^{2}-\frac{5}{2}\right]\right\}
$$

where species pressure $p_{s}$ is defined as $p_{s}=\rho_{s} k \hat{T} / m_{s}=n_{s} k \hat{T}$, Prandtl number $\operatorname{Pr}$ for the mixture and $G_{s}(\underline{u})$ is the equilibrium distribution function in the Groppi et al. model [8]:

$$
G_{s}(\underline{u})=n_{s}\left(\frac{m_{s}}{2 \pi k \hat{T}}\right)^{3 / 2} \exp \left[-\frac{m_{s}}{2 k \hat{T}}\left(\underline{u}-\underline{u}_{s}^{(g)}\right)^{2}\right]
$$


The Shakhov-based model modifies the distribution function by introducing a heat flux correction. For a mixture of gases the heat flux is an energy flux dependent on a coupling between the Fourier and the Duffour effects. The correction in the distribution function is only affected by the temperature gradient and it follows from the moment integration with respect to the species mean velocity. The heat flux correction obtained from the Chapman-Enskog expansion (with $\epsilon$ the small parameter related to Knudsen) becomes,

$$
\begin{gathered}
\underline{q}_{s}^{\text {corr }}=\int_{-\infty}^{\infty} m_{s}\left(\underline{u}-\underline{u}_{s}\right) \frac{1}{2}\left(\left(\underline{u}-\underline{u}_{s}\right)^{2}\right)\left\{G_{s}^{S h}-\frac{\epsilon}{v}\left(\frac{\partial f_{s}^{M}}{\partial t}+\underline{u} \cdot \frac{\partial f_{s}^{M}}{\partial \underline{x}}\right)\right\} d \underline{u} \\
\underline{q}_{s}^{\text {corr }}=-\frac{\epsilon}{v} \frac{5}{2} \frac{k}{\operatorname{Pr}} \frac{n_{s} k T}{m_{s}} \frac{\partial T}{\partial \underline{x}}+O\left(\epsilon^{2}\right)
\end{gathered}
$$

Since the Shakhov-based correction affects the distribution function and only high order moments, the expressions for $\underline{u}_{s}^{(g)}$ and $\hat{T}$ are unchanged in comparison to the original model [8].

$$
\underline{u}_{s}^{(g)}=\left(1-\frac{\eta}{v}\right) \underline{u}_{s}+\frac{\eta}{v} \underline{u}_{0}
$$

From the energy conservation, the modified temperature is in the form:

$$
\hat{T}=T-\frac{1}{3 n k} \sum_{s=1}^{2} \rho_{s}\left(\underline{u}_{s}^{(g)}-\underline{u}_{0}\right)^{2}=T-\frac{1}{3 n k}\left(1-\frac{\eta}{v}\right)^{2} \sum_{s=1}^{2} \rho_{s}\left(\underline{u}_{s}-\underline{u}_{0}\right)^{2}
$$

To preserve positive temperature fields the relaxation ratio is limited as $0 \leq \eta / v \leq 2$.

\section{ES-based Model}

In the second proposed kinetic model, an anisotropic modification is added, following the approach used for the ES model for a single-species monoatomic gas flow [18, 19]. The collision operator is modified to include the relaxation characteristic for the ES model. The governing relaxation equation is in the form:

$$
\frac{\partial f_{s}}{\partial t}+\underline{u} \cdot \frac{\partial f_{s}}{\partial \underline{x}}=Q_{s} \quad ; \quad Q_{s}=\frac{v}{1-v_{E S}}\left(G_{s}^{E S}-f_{s}\right) ; \quad ; \quad s=1,2
$$

The new distribution function has the form:

$$
G_{s}^{E S}(\underline{u})=\frac{n_{s}}{\sqrt{\operatorname{det}\left(2 \pi \underline{\Lambda}_{s}\right)}} \exp \left[-\frac{1}{2}\left(\underline{u}-\underline{u}_{s}^{(g)}\right) \cdot \underline{\Lambda}_{s}^{-1} \cdot\left(\underline{u}-\underline{u}_{s}^{(g)}\right)\right]
$$

The tensor $\underline{\Lambda}_{s}$ is introduced by the temperature $\hat{T}$ and the ES relaxation parameter $v_{E S}$ :

$$
\underline{\underline{\Lambda}}_{s}=v_{E S} \underline{\underline{\Theta}}_{s}+\left(1-v_{E S}\right)\left(\frac{k \hat{T}}{m_{s}}\right) \underline{I} \quad ; \quad \underline{\Theta}_{s}=\frac{1}{n_{s}} \int_{-\infty}^{\infty}\left(\underline{u}-\underline{u}_{s}^{(g)}\right) \otimes\left(\underline{u}-\underline{u}_{s}^{(g)}\right) f_{s} d \underline{u}
$$

The collision operator $Q_{s}$ is modified by dividing it with $\left(1-v_{E S}\right)$ which introduces changes in the species target velocity and temperature. A comparison with the BGK expression of $\hat{T}$ shows that the additional terms come from the relaxation change of the ES model with $v_{E S}$ and from the introduced drift velocity.

$$
\begin{aligned}
& \underline{u}_{S}^{(g)}=\left(1-\frac{\eta}{v}\left(1-v_{E S}\right)\right) \underline{u}_{s}+\frac{\eta}{v}\left(1-v_{E S}\right) \underline{u}_{0} \\
& \hat{T}=T-\frac{1}{3 n k}\left[\left(1-\frac{\eta}{v}\left(1-v_{E S}\right)\right)^{2} \sum_{s=1}^{2} \rho_{S}\left(\underline{u}_{S}-\underline{u}_{0}\right)^{2}\right]
\end{aligned}
$$

To guarantee positive temperature the fraction $\eta / v$ is in the limits $0 \leq \eta / v \leq 2 /\left(1-v_{E S}\right)$. In comparison to the Groppi and Shakhov-based models the upper boundary for the $\eta / v$ relaxation parameter is more restricted due to the introduction of $v_{E S}$ and has a maximum value of $\eta / v=4 / 3$ for $v_{E S}=-0.5$. 


\section{NUMERICAL APPLICATION}

\section{Normal Shock Wave}

The numerical evaluation of the models begins with an investigation of the structure of a normal shock wave. The setup of the problem is as follows: a binary gas mixture flow, with defined species concentration and mass ratio, undergoes strong non-equilibrium changes through a normal shock wave to arrive at a different equilibrium state. The state to state change is initialized with the Rankine-Hugoniot conditions for the macroscopic variables and Maxwellian distributions are set at the two different initial equilibrium states. The discrete velocity method (DVM) [13, 14] is used to apply the two kinetic models. For the one-dimensional normal shock wave a hundred uniformly spaced discrete velocities are used and are sufficient. A more detailed study of this problem is presented in [12] and one of the investigated shock profiles is shown here for completeness.

On Fig. 1 we examine the flow at free-stream Mach number 3, mass ratio between species 2, and concentration of the light gas (species 1) - 90 percent. An example setup of a mixture of noble gases with that mass ratio is the mixture of neon and argon. A viscosity law with $\omega=0.5$ is used for a hard-sphere mixture model. We evaluate the models by comparing the normalized species number density $n_{1}$ and $n_{2}$ (Fig. 1 (a)) and species temperatures $T_{1}$ and $T_{2}$ (Fig. 1 (b)) through the shock. We validate against the results from the full Boltzmann equation [15], which are presented by the red elements- squares for species 1 and triangles for species 2 . The figures show in solid (species 1 ) and dashed (species 2) black lines, how well the Groppi et al.'s model follows the Boltzmann results for the structure of the shock wave. The model is simulated by setting the Prandtl number to 1 in the Shakhov-based solver or $v_{E S}=0$ in the ES-based solver. This eliminates the Shakhov-based or ES-based correction, respectively, and demonstrates the results that can be obtained by the original - Groppi et al.'s model. The figures also show the results obtained by the Shakhov-based model in blue and ES-based model in green. The improvement of the slope for the species number density on Fig. 1 (a) is evident, but small for both models. However, the species temperature deviates significantly from the Groppi et al.'s model and follows much more closely the results of the full Boltzmann equation. This means that the significance of the two corrected models increases for higher order moments. We also notice that as every BGK-based model, upstream of the shock the models have longer tails than the Boltzmann results suggest and the models also deviate from each other. If we compare the two models, the Shakhov-based model performs slightly better upstream of the shock, which is consistent with studies evaluating the Shakhov and ES models for a single species gas [20].

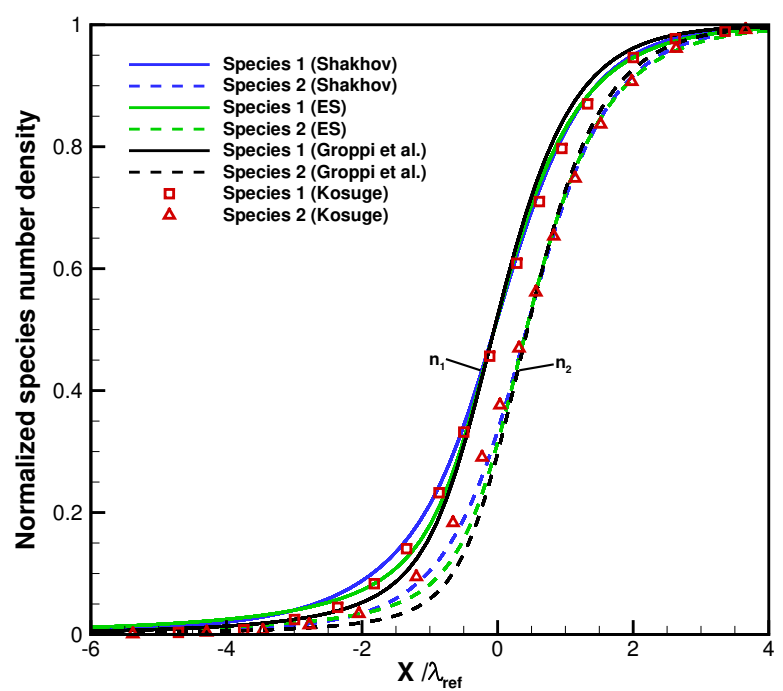

(a) Species number density

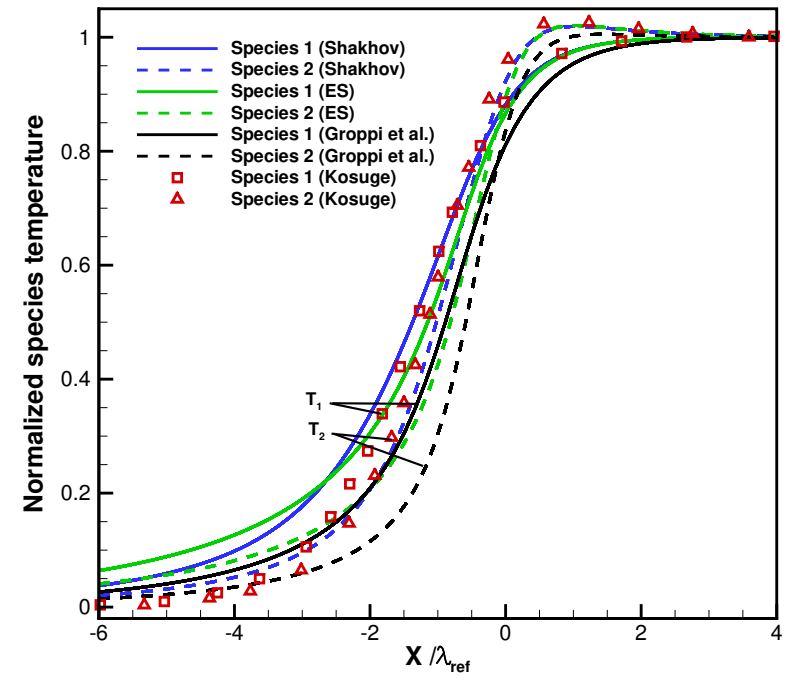

(b) Species temperature

FIGURE 1. Profile of a normal shock wave - validation and comparison between the model by Groppi et al.'s, Shakhov- and ES-based models with Kosuge's full Boltzmann model [15] for free-stream Mach number 3 [12].

The two models introduce changes that lead to a correct Prandtl number and in the continuum limit a third 
transport coefficient- the thermal conductivity. This affects all variables, defining the flow, but mostly the heat flux. In the present work we demonstrate the difference of the heat flux prediction between the Groppi et al.'s, Shakhov-based and ES-based models through the normal shock. Figure 2 inspects the total heat flux of the same test case for freestream Mach number 3, mass ratio of 2 and 90 percent light species concentration. On the plot we see the variation of the non-dimensionalized heat flux through the normal shock. As expected, the heat flux changes through the shock and is negative, since the thermal conduction effect is stronger than the Duffour effect. Note that the ES-based model has a longer upstream tail as in the species temperature result. We observe a difference between the two new models of 4.1 percent, while the Shakhov-based and Groppi et al.'s models differ by a total of 20.5 percent. This emphasizes the importance of fitting correctly the transport coefficients and in particular the thermal conductivity.

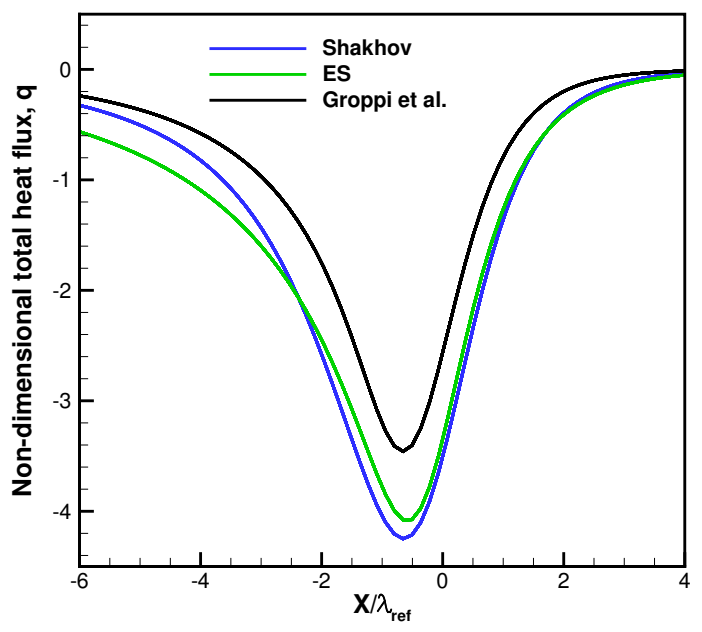

FIGURE 2. Non-dimensional total heat flux, q for the Shakhov-based, ES-based and Groppi models through a normal shock wave with free stream Mach number 3.

\section{Cylinder}

A more complex test case - a two-dimensional flow with $48 \times 48$ and $64 x 64$ (for the higher mass ratio) uniformly spaced discrete velocities- is investigated. The same free-stream conditions as for the normal shock wave are applied for the supersonic flow over a circular cylinder. A bow shock forms ahead of the cylinder. On Fig. 3 and 4 a flow with free-stream Mach number 3, Knudsen number 0.1, based on the radius of the cylinder and light species concentration 90 percent of the gas is presented. We know from our previous simulations that the biggest differences between species occur at regions with strong non-equilibrium effects. We also know that the lighter species (species 1) reacts faster to those changes and then the heavier gas (species 2) has to catch up until they find a balance. This is where the bow shock that the cylinder causes is placed. On Fig. 3 the variation between species mean velocity is shown, while Fig. 4 focuses on the variation between species temperature. In the vicinity of the shock, the velocity of the lighter gas $u_{1}$ starts decreasing first and the difference between the two species $u_{2}-u_{1}$ increases, until species 2 catches up post shock. Unlike the velocities, the heavy species temperature has a steeper slope, increases faster and crosses the lighter species temperature in the shock wave. This we can clearly observe in the plots. Temperature $T_{1}$ increases first, creating a negative $T_{2}-T_{1}$ difference in blue and then $T_{2}$ catches up and surpasses $T_{1}$ leading to a positive temperature difference $T_{2}-T_{1}$ in red.

Each figure contains three plots with varied conditions. On Fig. 3 (a) and (b) and Fig. 4 (a) and (b) the mass ratio between species is 2 and the assigned wall temperature is changed from $T_{\text {wall }}=1.5 T_{\infty}$ in (a) to $T_{\text {wall }}=2.5 T_{\infty}$ in (b) to inspect the dependency of the flow. The non-equilibrium effect in the flow through the bow shock, expressed by the difference in species velocities and temperatures, is not affected. For the considered wall temperatures, the shock stand-off distance is not significantly changed. 


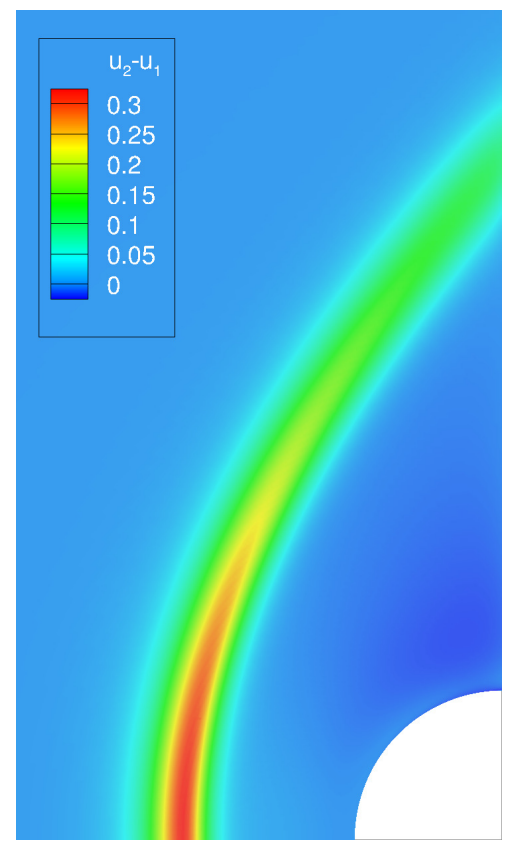

(a) $m_{2} / m_{1}=2, T_{\text {wall }}=1.5 T_{\infty}$

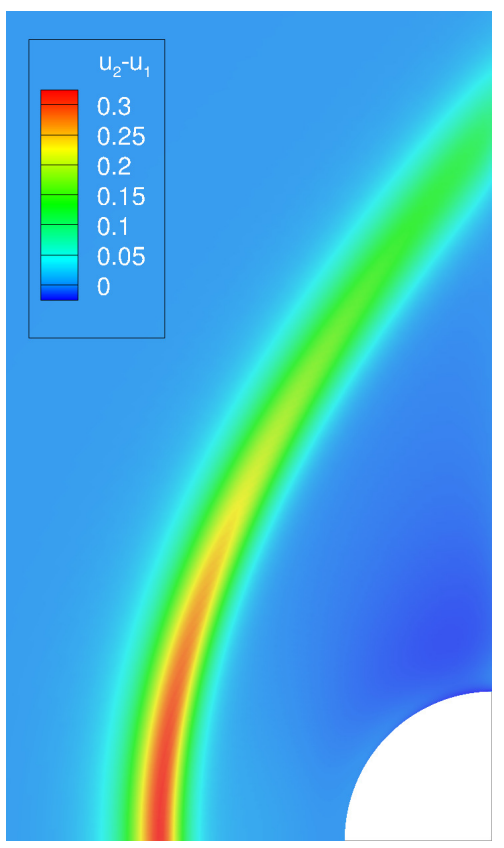

(b) $m_{2} / m_{1}=2, T_{\text {wall }}=2.5 T_{\infty}$

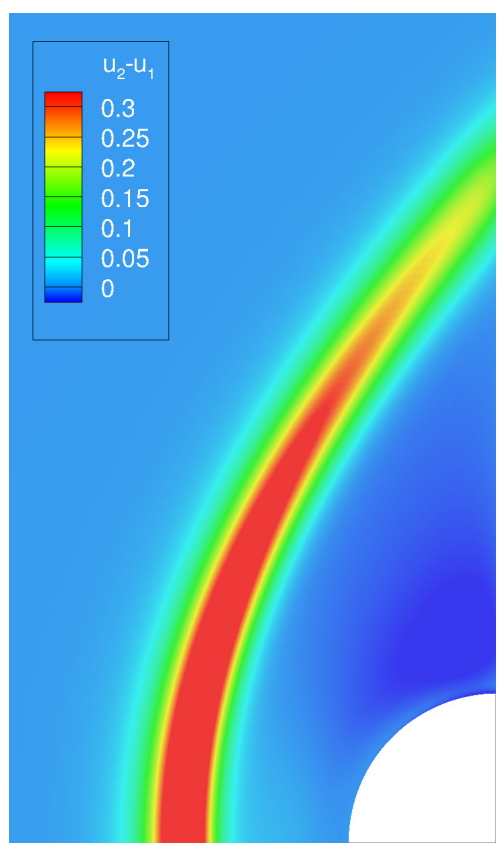

(c) $m_{2} / m_{1}=4, T_{\text {wall }}=1.5 T_{\infty}$

FIGURE 3. Non-dimensional species velocity drift in a binary mixture flow over a cylinder (velocity is non-dimensionalized with the most probable speed of the lighter gas). Results were obtained with the Shakhov-based model.

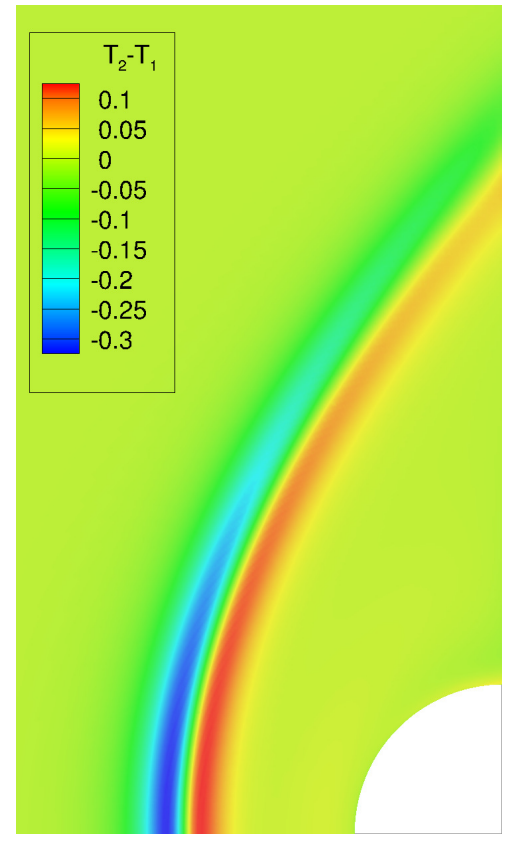

(a) $m_{2} / m_{1}=2, T_{\text {wall }}=1.5 T_{\infty}$

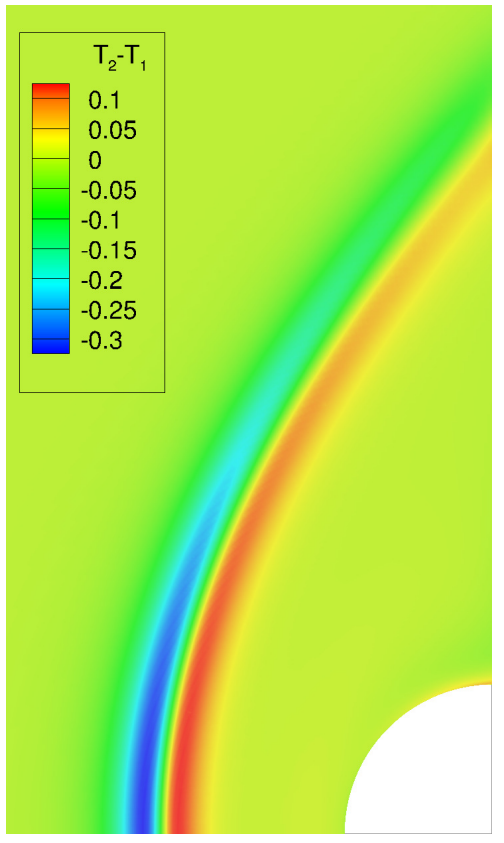

(b) $m_{2} / m_{1}=2, T_{\text {wall }}=2.5 T_{\infty}$

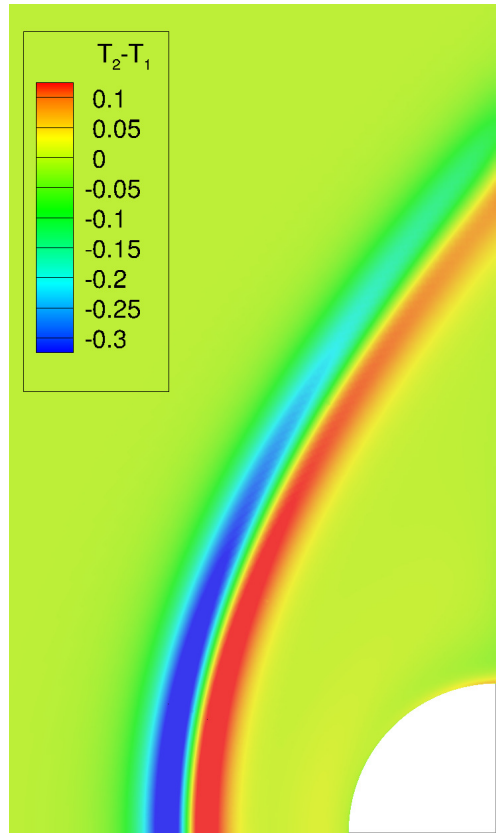

(c) $m_{2} / m_{1}=4, T_{\text {wall }}=1.5 T_{\infty}$

FIGURE 4. Non-dimensional species temperature drift in a binary mixture flow over a cylinder. Results were obtained with the Shakhov-based model. 
Changing the mass ratio between species to 4 , however, affects the species mean velocities and temperatures significantly, as we can see on Fig. 3 (c) and 4 (c). For the higher mass ratio, the non-equilibrium effects and species differences are stronger. The same is observed for the species temperature drift.

Until now we were focused on emphasizing the difference between species mean properties as a way of establishing the non-equilibrium of the flow. The discussed results were obtained with the Shakhov-based kinetic model. We now consider the stagnation streamline (the streamline on which the stagnation point lies) for the same two cylindrical cases - flows with free-stream Mach number 3, Knudsen number 0.1 and a mass ratio of 2 and 4 . The goal of this study is to compare the Shakhov- and the ES-based models with DSMC results. The DSMC solver used in this work is dsmcFoam+ [16]. To remain consistent with the kinetic models, the Knudsen number $(K n=0.1)$ is based on the free-stream mean free path of the lighter gas species and the cylinder radius, and the Mach number $\left(M_{\infty}=3\right)$ is based on the gas constant of the mixture. In both cases, the light species is neon, with argon and krypton being used for the mass ratios of 2 and 4, respectively. The variable hard sphere collision model is used $(\omega=0.72)$, with fully diffusive reflections considered for the cylinder surface interactions. As before, the cylinder wall temperature is held constant at $1.5 T_{\infty}$. Each simulation contained around 2.7 million DSMC simulator particles at steady state. The number of samples at steady state for the mass ratio of 4 are 50,000 and for the mass ratio of $2-100,000$ samples.

The DSMC results are shown in black lines, while the Shakhov-based and ES-based solutions are in blue and green respectively.We plot the non-dimensional number densities and temperatures through the shock and until the solid is reached at $x / R=-1$, where $R$ is the radius of the cylinder. On Fig. 5 the non-dimensionalized total number density $n$ and the heavy gas number density $n_{2}$ are shown. Physically, the gas undergoes a compression effect through the shock and then towards the stagnation point of the cylinder, demonstrated by the increase in number density. Figure 5 shows good agreement in the stagnation streamline density profiles predicted by dsmcFoam + and the kinetic models, with the ES-based model slightly closer to the DSMC than the Shakhov-based model.

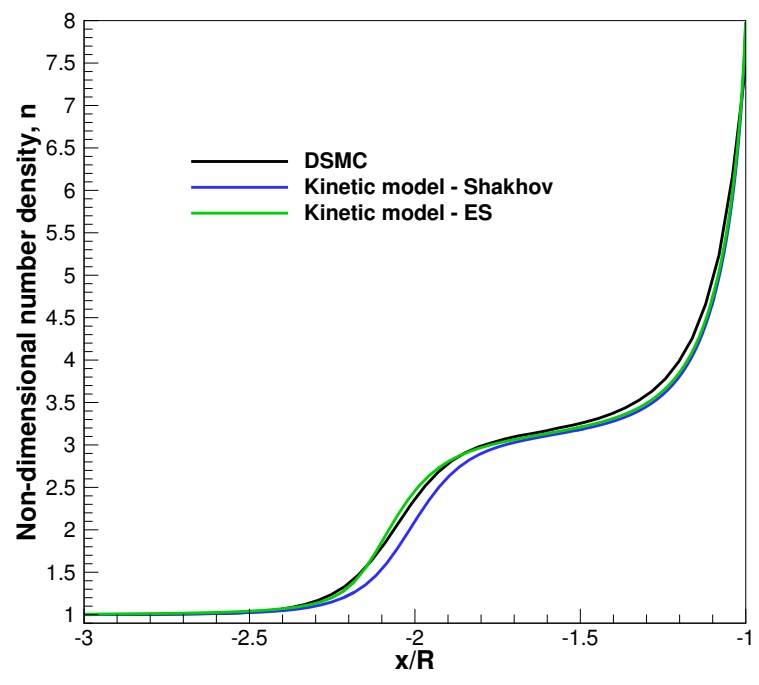

(a) Total number density

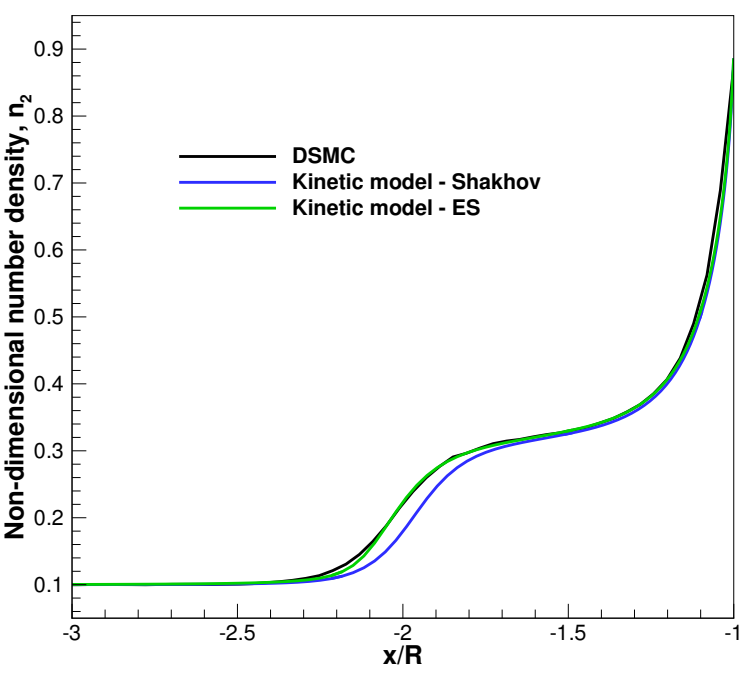

(b) Heavier-species number density

FIGURE 5. Comparison of the total and heavy species number densities for the Shakhov and ES kinetic models with DSMC results for a mass ratio of 2 .

The mixture temperature is shown on Fig. 6 (a). The temperature jump is caused by the shock and later on relaxes, going into the boundary layer of the cylinder towards the assigned wall temperature $T_{\text {wall }}=1.5 T_{\infty}$. The figure shows a reasonable agreement in the stagnation streamline temperature profile for the gas mixture, with DSMC predicting a slightly larger shock stand off distance in comparison to the two kinetic models and a slightly lower peak temperature than the Shakhov-based model.

It is very interesting to observe the variation between species temperature $T_{2}-T_{1}$ (Fig. 6 (b)). As in the normal shock study, the increase in $T_{1}$ is faster and therefore negative difference between the temperatures $T_{2}-T_{1}$ is seen in the upstream of the shock. Then a steeper growth of the heavy species temperature, creates a positive $T_{2}-T_{1}$ difference, until we reach equilibrium, where the species temperatures are equalized. We see that the kinetic models 


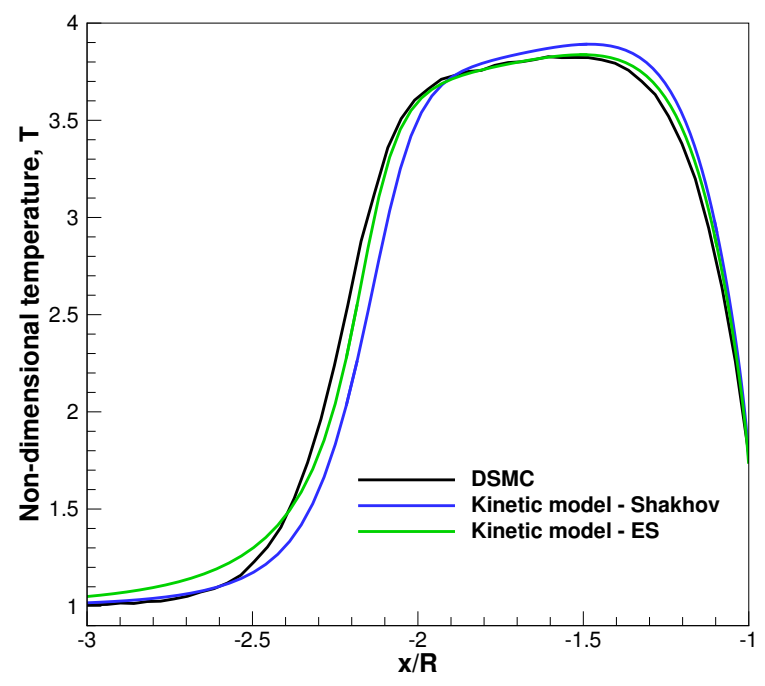

(a) Mixture temperature

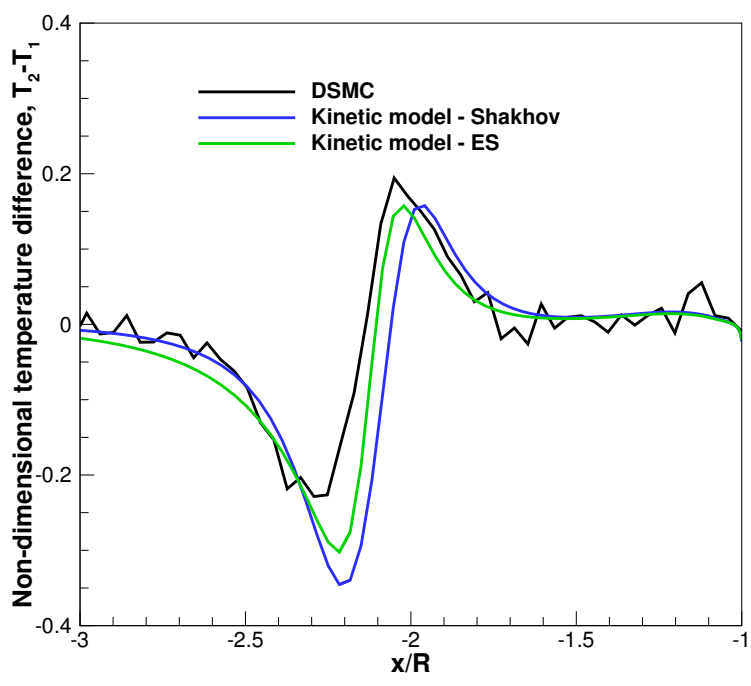

(b) Species-temperature difference

FIGURE 6. Comparison of the mixture temperature and the species temperature drift for the Shakhov and ES kinetic models with DSMC results for a mass ratio of 2 .

follow qualitatively the results by DSMC, but the increase of the temperature of the light species is bigger. The DSMC results display some statistical scatter, since the heavy gas species is only $10 \%$ of the gas mixture and therefore a trace species in the DSMC simulation. This makes reducing the statistical scatter challenging with the use of constant particle weighting factors. Here we observe one of the benefits and motivations of kinetic modelling - the lack of statistical scatter for similar problems.

Considering the higher mass ratio of 4 (neon-krypton gas mixture), Fig. 7 shows very good agreement in the stagnation streamline density profiles predicted by dsmcFoam + and the kinetic models.

Figure 8 (a) shows reasonable agreement in the stagnation streamline temperature profile for the gas mixture. The shock stand off difference between the models and DSMC is reduced and the peak temperature is preserved better as compared to the lower mass ratio.

The difference between species temperatures in Fig. 8 (b) is well captured by the kinetic models and DSMC in its decrease, while the increase predicted by dsmcFoam + has a significantly greater peak temperature difference than the kinetic models. The DSMC results do not exhibit as much scatter as the lower mass ratio, despite using less statistical samples, because the non-dimensional temperature differences between the species are greater at the higher mass ratio. For the higher mass ratio, the two kinetic models are fairly close to each other, while the Shakhovbased model is matching the DSMC results slightly better than the ES-based model. Overall, the comparison between Shakhov-based, ES-based and DSMC shows good agreement, but further inspection of different parameters like Mach number and concentration are required. This will be considered in future work. 


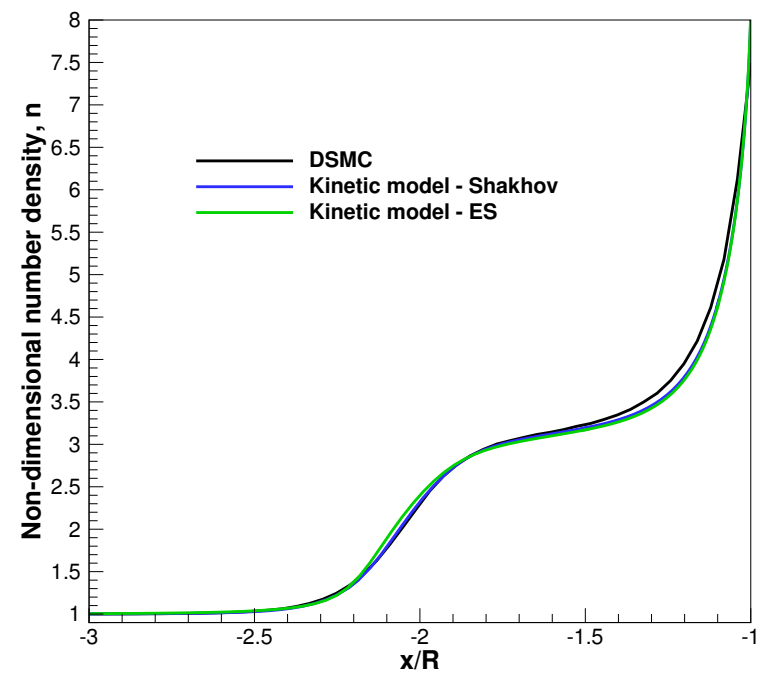

(a) Total number density

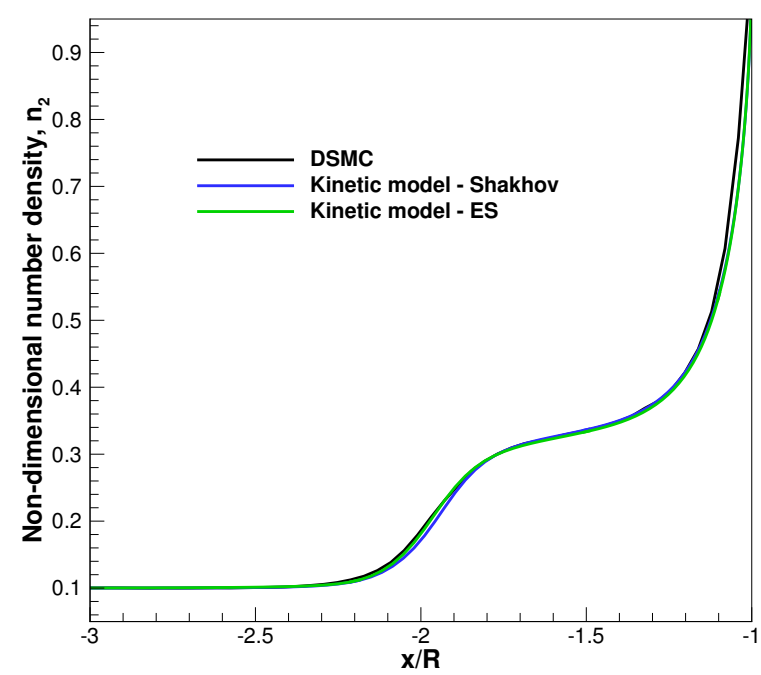

(b) Heavier-species number density

FIGURE 7. Comparison of the total and heavy species number densities for the Shakhov and ES kinetic models with DSMC results for a mass ratio of 4 .

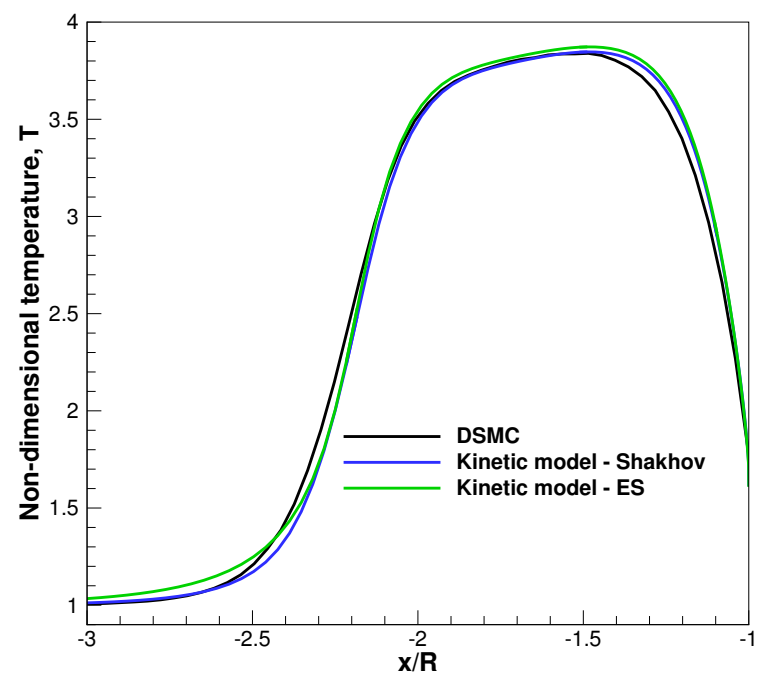

(a) Mixture temperature

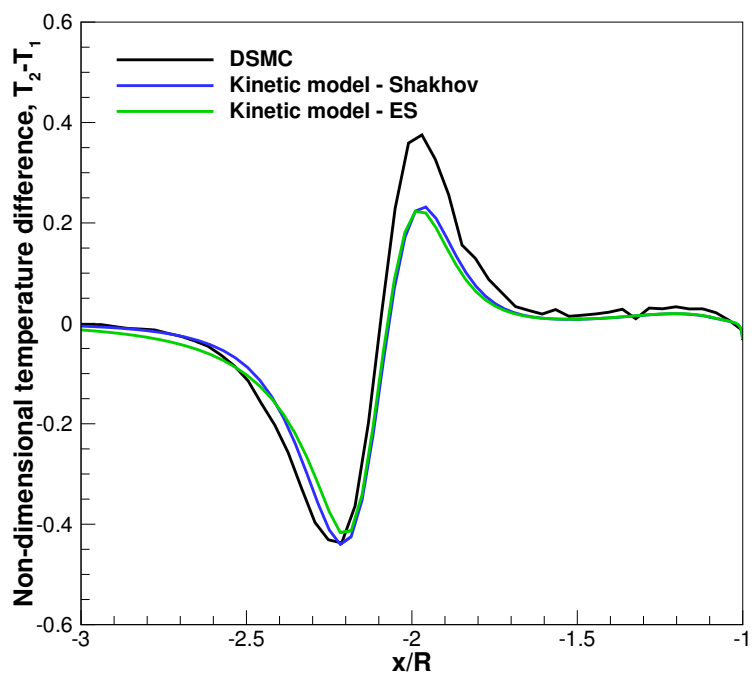

(b) Species-temperature difference

FIGURE 8. Comparison of the mixture temperature and the species temperature drift for the Shakhov and ES kinetic models with DSMC results for a mass ratio of 4 . 


\section{CONCLUSION}

Two newly introduced kinetic models were tested and compared through simulations examining the structure of a normal shock wave and the Mach 3 flow over a cylinder. The comparisons with the benchmark results for the full Boltzmann equation and DSMC respectively, show promising results from both models for a range of species mass ratios and imposed wall temperature in the cylinder cases. In future work, further comparison with DSMC results will be presented. Furthermore, the proposed kinetic models will be used as basis for gas kinetic schemes, offering higher computational efficiency for flows at moderate levels of thermodynamic non-equilibrium. Also, the extension to diatomic gas flows will be considered.

\section{ACKNOWLEDGMENTS}

The authors would like to acknowledge the financial support provided by the University of Glasgow. Results were obtained using the EPSRC funded ARCHIE-WeSt High Performance Computer (www.archie-west.ac.uk). EPSRC grant no. EP/K000586/1.

\section{REFERENCES}

[1] S. Chapman and T. Cowling, The Mathematical Theory of Non-Uniform Gases, 2nd ed. (Cambridge University Press,London, 1952).

[2] G. Bird, Molecular Gas Dynamics and the Direct Simulation of Gas Flows (Oxford Science Publications, 1994).

[3] R. Wang and K. Xu, AIP Conference Proceedings 1628, 970-975 (2014).

[4] Y. Zhang, L. Zhu, R. Wang, and Z. Guo, Phys. Rev. E 97, p. 053306 (2018).

[5] P. Andries, K. Aoki, and B. Perthame, Journal of Statistical Physics 106, 993-1018 (2002).

[6] S. Brull, V. Pavan, and J. Schneider, European Journal of Mechanics - B/Fluids 33, 74 - 86 (2012).

[7] S. Brull, Communications in Mathematical Sciences 13, 1 - 13 (2015).

[8] M. Groppi, S. Monica, and G. Spiga, EPL (Europhysics Letters) 96, p. 64002 (2011).

[9] C. Klingenberg, M. Pirner, and G. Puppo, Kinetic \& Related Models 10, 445-465 (2017).

[10] C. Klingenberg and M. Pirner, Journal of Differential Equations 264, 702-727 (2018).

[11] J. R. Haack, C. D. Hauck, and M. S. Murillo, Journal of Statistical Physics 168, 826-856 (2017).

[12] B. Todorova and R. Steijl, European Journal of Mechanics - B/Fluids (accepted) (2019).

[13] R. Steijl and G. Barakos, International Journal for Numerical Methods in Fluids 62, 1081-1106 (2010).

[14] S. Colonia, R. Steijl, and G. Barakos, AIAA Journal 54, 1264-1276April (2016).

[15] S. Kosuge, K. Aoki, and S. Takata, European Journal of Mechanics - B/Fluids 20, 87 - 126 (2001).

[16] C. White, M. Borg, T. Scanlon, S. Longshaw, B. John, D. Emerson, and J. Reese, Computer Physics Communications 224, 22 - 43 (2018).

[17] E. M. Shakhov, Fluid Dynamics 3, 95-96 (1968).

[18] L. H. Holway, Jr., Rarefied Gas Dynamics, Volume 1, 193-215 (1965).

[19] P. Andries, P. L. Tallec, J. Perlat, and B. Perthame, European Journal of Mechanics - B/Fluids 19, 813 - 830 (2000).

[20] S. Chen, K. Xu, and Q. Cai, Advances in Applied Mathematics and Mechanics 7, 245-266 (2015). 